\title{
Quantification of Protective Antibody Vaccine-Elicited in Chickens against Infectious Bursal Disease Virus
}

\author{
Mohammad Nazrul Islam ${ }^{1}$, Md. Mostafizer Rahman ${ }^{1}$, Mir Rowshan Akter ${ }^{1}$, Md. Shahidur Rahman Khan ${ }^{2}$, Md. \\ Salauddin $^{1}$ and Md. Hemayatul Islam ${ }^{3}$ \\ 1. Department of Microbiology, Faculty of Veterinary and Animal Science, Hajee Mohammad Danesh Science and Technology \\ University, Dinajpur-5200, Bangladesh \\ 2. Department of Microbiology \& Hygiene, Bangladesh Agricultural University, Mymensingh-2202, Bangladesh \\ 3. Department of Veterinary and Animal Sciences, University of Rajshahi, Rajsahahi-6205, Bangladesh
}

\begin{abstract}
A study on infectious bursal disease virus (IBDV) on chickens of Cobb-500 strain broiler breed at Thakurgaon district of Bangladesh was performed. The protective antibody was measured on one day old chicks (DOC) and post-vaccinated (PV) flocks up to 75 weeks by indirect enzyme linked-immunosorbent assay (I-ELISA). The assays have included five flocks with vaccination historic against IBDV contained 45,000 birds with age ranging from DOC to 75 weeks, just before culling. Maternally derived antibody (MDA) mean titer (MT) ranged from 3,395 to 5,184. The antibody from serum samples ( $=92$ per flock) were titer tested by I-ELISA at age $14 \mathrm{~d}, 5,8,23,50$ and 75 weeks of each vaccinated flock. Antibody titer level gradually decreased before vaccination. Vaccination done by intermediate plus vaccine resulting titers level was increased and stayed at the same level. The antibody MT at the 14th day was 500, which supported Deventer method. The protective antibody MT was declined at growing, laying, mid laying and last stage of laying groups. So MDA titer was enough in offspring that could protect birds easily.
\end{abstract}

Key words: Infectious bursal disease (IBD), broiler breeder of strain Cobb-500, antibody titer, maternally derived antibody (MDA), indirect ELISA.

\section{Introduction}

Infectious bursal disease (IBD) is an important viral disease of poultry throughout the world [1]. IBD is an acute, highly contagious viral disease of young chicken and it affects the poultry industries worldwide [2]. This disease in poultry which causes heavy economic losses due to immunosuppression in case of subclinical cases [3] and in acute, it is associated with mortalities, hemorrhages with bursa damage [4]. The acute form of clinical signs characterized by sudden unusual calmness in a jubilant flock, vent picking, body tremor, paralysis of both legs, stretched backward with yellowish watery diarrhea, depression, anorexia, prostration and finally death [5]. Chickens have a short incubation period and acute cases

Corresponding author: Mir Rowshan Akter, associate professor, research field: molecular bacteriology. characterized by high morbidity and variable mortality. Poultry sector has a tremendous employment generating opportunity in reducing unemployment problem in Bangladesh and other countries of world. Poultry meat now accounts for more than $30 \%$ of all meat consumed in Bangladesh. The world's average annual per capita poultry meat consumption is currently $9.5 \mathrm{~kg}$ [6]. On the other hand, the growth of this profitable sector is interrupted by a number of infectious and contagious diseases like Newcastle disease, Gumboro disease, infectious bronchitis, Collibacillosis, salmonellosis, fowl cholera, avian influenza and mycoplasmosis. Moreover, IBD is one of the important diseases, cause heavy economic loss among them. The poultry industry is now in a great challenge to IBD as it appears as emerging and fatal disease throughout the world as like as Bangladesh. Hemorrhage is found in breast and thigh muscles but 
not always present. Also abnormally swollen liver and kidney, especially kidney full of urinates, most prominently swollen edematous bursa of fabricus from mild to profuse hemorrhage is found in IBD during postmortem examination. Cho and Edgar [7] described the signs of IBD as well as pathological changes occurring in lymphoid organs and observed that peak mortality occurred at 4-6 d after onset of the disease. Giambrone [8] stated that IBD is subclinical form was associated with a variant IBD virus (IBDV) which varied in ability to cause mortality but invariably caused immunosuppression. World Poultry conducted a survey with the help of international poultry specialist which focus on continuing sanitary status in the poultry sector. Gumboro disease topped the list of the most serious poultry diseases [9].

Therefore, the present study was designed i) to determine maternally derived IBD antibody titer level in day old chicks (DOC) of different broiler breeder; ii) to determine serum antibody titer level on different age groups of broiler breeder against IBD and recommend vaccination schedule based on titer level.

\section{Materials and Methods}

The IBDV infection was surveyed in Akhanagar private poultry farm of Thakurgaon district in Rangpur division, Bangladesh. A questionnaire survey was made to estimate the population, strains, diseases, mortality, flock size, vaccination, etc. to select the broiler breeder flock for study. Five broiler breeder flocks were selected rearing about 9,000 birds/flock from DOC up to 75 weeks.

\subsection{Sample Collection}

Blood sample from sacrificed of DOC $(\mathrm{N}=25)$ from each flock was kept directly from the heart using sterile plastic disposable $3 \mathrm{~mL}$ syringe. As per design of the study blood sample $(\mathrm{N}=92)$ were collected from each flock chickens on the age of $14 \mathrm{~d}, 5,8,23$, 50 and 75 weeks. The blood was collected from wing vein (Fig. 1) by $3 \mathrm{~mL}$ sterile plastic disposable syringe.
All sera have harvested in both essays were aliquoted in Eppendorf tubes previously identified stocked at $-20{ }^{\circ} \mathrm{C}$ until use. The protective antibody titer of each serum was measured by indirect enzyme linked-immunosorbent assay (I-ELISA).

\subsection{Indirect Enzyme Linked-Immunosorbent Assay (I-ELISA)}

One single serum dilution (1:500) was applied for the detection of maternally derived antibody (MDA) of IBDV specific antibody. The test performed based on IBD ELISA Kit manufacturer, Biochek, Holland. Diluted samples were placed on IBD antigen coated test plate. Conjugate placed on IBD antigen coated test plate. Then micro titer plate showed color (yellowish) development in I-ELISA (Figs. 1-4).

\subsection{Calculation of Results}

The IBD antibody positive control was standardized. The IBD antibodies titer in chicken samples was figured out in the relationship with the positive control: expressed as $\mathrm{S} / \mathrm{P}$ ( $\mathrm{S}=$ sample; $\mathrm{P}$ = positive ratio). The test was validated when the mean negative control absorbance was below 0.3 and the difference between the mean negative control and the mean positive control was greater than 0.15 .

Interpretation of results:

Sample with an S/P of 0.2 or greater contain anti-IBD antibodies and were considered positive.

(1) Mean of negative control: well A1 + well B1/2 (Figs. 2 and 4);

(2) Mean of positive control: well C1 + well D1/2 (Figs. 2 and 4);

(3) Calculation of S/P ratio: (mean of test sample mean of negative control)/(mean of positive control mean of negative control);

(4) Calculation of antibody titer: the following equation relates the $\mathrm{S} / \mathrm{P}$ of a sample at a 1:500 dilution to an end point titer.

$$
\log 10 \text { titer }=1.1 * \log (\mathrm{SP})+3.361
$$




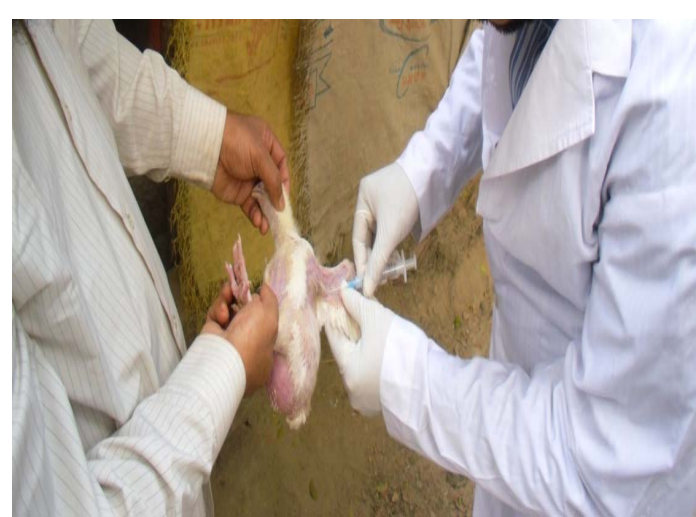

Fig. 1 Collection of blood from wing vein.

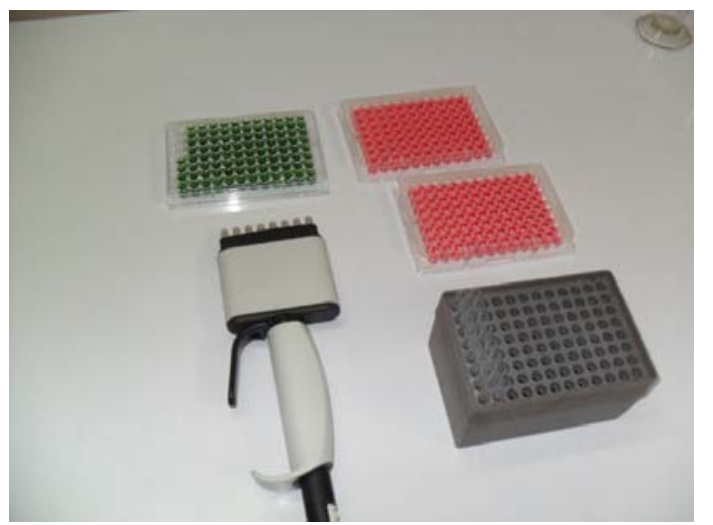

Fig. 3 Conjugate placed on IBD antigen coated test plate.

\subsection{Calculation of Antibody Titer Using Computer Software Program Developed of Biochek}

This software contained data (positive control, negative control, S/P ratio and optical density (OD) value) which were adjusted in such a way that when OD value of the samples that were obtained from ELISA reader are fed, the antibody titer of the samples obtained automatically. To get the antibody titer of all 92 samples in the particular plate, positive control and negative control should be kept fixed in the program only for that particular plate.

The optimal vaccination time for IBD was calculated by the Deventer formula [10] as follows:

Vaccination age $=[(\log 2$ IBDV antibody titer of the bird (\%) - log2 ELISA breakthrough titer of the vaccine) $\left.\times t^{1 / 2}\right]+$ age at sampling + correcting value $0-4$

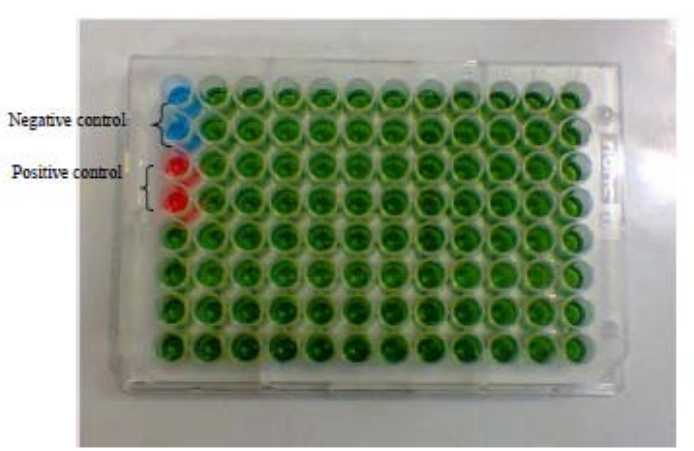

Fig. 2 Diluted sample placed on infectious bursal disease (IBD) antigen coated test plate.

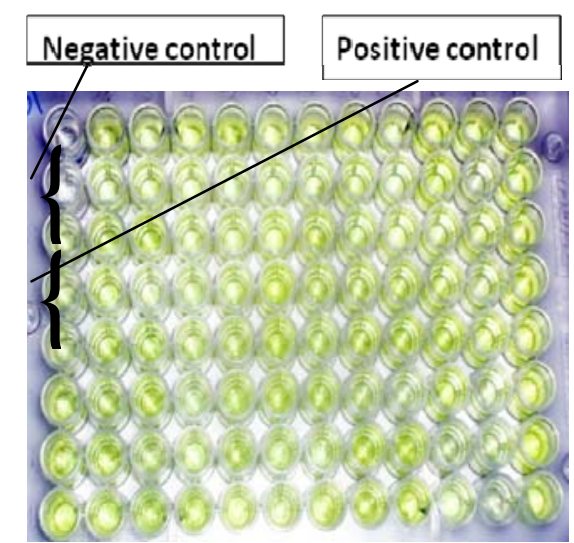

Fig. 4 Micro titer plate showing color development in indirect enzyme linked-immunosorbent assay (I-ELISA).

where bird (\%): titer of the bird (at sampling) that represents certain percentage of the flock (in this study: $75 \%$ ) was used. The highest $25 \%$ was rejected and the highest titer from the remaining was used. Breakthrough: breakthrough (ELISA) titer of the vaccine to be used (live intermediate IBD vaccine with breakthrough titer of 125 was used). $t^{1} \frac{1}{2}$ : half life time of the antibodies (ELISA titer) in the type of chickens that were sampled (dual purpose breeds: $3.5 \mathrm{~d}$ ). Age at sampling: age of the birds at sampling. It was two. Correcting value $0-4$ : extra days when the sampling was done at 0-4 $\mathrm{d}$ post hatch.

\subsection{Data Analysis by Software}

As in each antigen coated plate two positive control, two negative control, 92 serum samples of each flock total 96 well was used, the OD value also imputed in 


\section{Quantification of Protective Antibody Vaccine-Elicited in Chickens against Infectious Bursal Disease Virus}

the software layout, respectively, then the antibody titer level generate automatically.

\section{Results}

The present study was conducted on broiler breeder tested by I-ELISA to determine MDA at age one day old against IBDV, to determine the titer level at different age groups of post vaccinated chicken and to predict vaccination time by showing persistence of antibody. The broiler breeder was vaccinated with intermediate plus 228E, intermediate type vaccine D78 \& 4way (combined formulation of IBD, Reo, ND and IB vaccine known as 4way vaccine) killed vaccine. The test was conducted on five flocks about 45,000 birds from DOC to 75 weeks. MDA mean titer (MT) of DOC was found MT ranged from 3,395 to 5,184 and vaccination prediction date $14^{\text {th }}$ to $16^{\text {th }}$ day after hatch, respectively. Vaccination date prediction was applied with the Deventer method predicting for an intermediate plus vaccine to vaccinate at $14 \mathrm{~d}$ of age (optimal titer to vaccinate $=500$, half-life of maternal derived antibody $=4.5 \mathrm{~d}$ and vaccination cover $=$ $75 \%)$.

\subsection{The IBDV MDA Titration}

From Table 1, the MDA MT at DOC were found MT highest and lowest value 5,184 and 3,395 (Table 1) and vaccination prediction date $14^{\text {th }}$ to $16^{\text {th }}$ day after hatch, respectively. Vaccination date prediction was performed with the Deventer method. Calculate the intermediate plus vaccine age at $14 \mathrm{~d}$ (optimal titer to vaccinate $=500$, half-life $\mathrm{MDA}=4.5 \mathrm{~d}$ and vaccination cover $=75 \%$ ).

\subsection{Measurement of Pre-vaccination Titer Level at} Age of $14 d$

Measurement of pre-vaccination titer level at age of $14 \mathrm{~d}$ is shown in Table 2. The highest titer level of MDA at day one was 614 (G.T.M). Drastically fall the MDA level in broiler breeder Cobb-500 at the age of $14 \mathrm{~d}$, after vaccination by intermediate plus vaccine the level optimum and protective.

3.3 Measurement of Post-vaccination IBDV Antibody Titer

Post vaccination titer level at age of five weeks by intermediate plus 228E \& intermediate D78 were 8,539 and 11,013 MT, respectively (Table 3). It might be protecting against IBD. The antibody titer level of broiler breeder Cobb-500 at the age of 8, 23, 50 and 75 weeks of the titer level was enough to produce high MDA for their offspring. In growing age the highest and lowest MT values were 11,013 and 8,539 (Table 3) but at the laying stage, these MT values were 7,250 \& 8,829 .

\subsection{Average MT at Different Ages in Bar Diagram}

The bar diagram (Fig. 5) shows that at age of five weeks the average MT of five flocks was the highest $(10,119)$, which was good enough for the day one for determining the optimum time for administration of live intermediate vaccine of IBD by Deventer formula [10]. Fig. 5 also showed that the MT value at eight weeks $(7,769), 50$ weeks $(7,725)$ and last stage just before culling 75 weeks $(6,495)$ was acceptable (> $6,000)$ to produce enough MDA for their offspring.

\subsection{Post Vaccination Titer Level at the Age Eight Weeks}

Effect of post vaccination titer level at the age eight weeks was present in Table 4. The antibody titer level of broiler breeder Cobb-500 at the age of eight weeks was also protective against IBD.

\subsection{IBD Antibody Titer Level at 23 Weeks of Age Groups}

The highest \& lowest IBD antibody titer at the age of 23 weeks was 10,733 and 7,165 (Table 5) which is enough to produce good MDA for offspring.

\subsection{IBD Antibody Titer Level at 50 Weeks of Age Groups}

The highest \& lowest IBD antibody titer at the age 
of 50 weeks was 8,829 and 7,250 (Table 6) which is enough to produce good MDA for offspring.

\subsection{IBD Antibody Titer Level at 75 Weeks of Age Groups}

According to Table 7 , the MT is declined $(4,783)$ at last stage of lying (just before culling) but the titer level was enough to produce protective MDA for their offspring.

\section{Discussion}

The virus affects the bursa of fabricius which plays a vital role in encoding and maturing immature lymphocytes so as to produce antibodies. At DOC the MDA titer MT value was gradually decreased (500) at the $14^{\text {th }}$ day (just before 1 st vaccination) which was the optimal titer to vaccinate. In agreement with Shrestha et al. [6] that showed the chicks vaccinated at around day 14 got significant level of antibody titer at age of day 29. On the other hand, Jung et al. [11] mentioned that the half-life of ELISA MDA ranges from $4.2 \mathrm{~d}$ to 12 d. Saijo and Higashihara [12] found that the half-life of MDA to IBD in chick was $3.46 \mathrm{~d}$. Al-Natour et al. [13] found that medium level of maternal antibody protects the progeny up to 7-14 $\mathrm{d}$ of age. Azab et al. [14] found that maternal antibody lasted $14 \mathrm{~d}$ in local chicken. Wisniewska and Stosik [15] found that MDA lasted until 11-19 $\mathrm{d}$ and sometimes $23 \mathrm{~d}$ after hatching. Current study indicated that the MDA level promptly rising at the age of five weeks treated by intermediate plus 228E \& intermediate D78, respectively. The titer was well protective against IBD. Regarding the study $1^{\text {st }}$ vaccination had done at the age of $14 \mathrm{~d}$ when MDA apparently low and the boostering at age of $21 \mathrm{~d}$. It is also clear that the birds are most susceptible to IBDV around 30-35 $d$ of age, especially in the absence of the MDA. Since MDA can potentially neutralize the vaccine if done at a very younger age, the first vaccination may be most effective if done at the 14th day via the drinking water. This would allow the maternal levels to degrade such that the vaccine would induce an effective protective immune response. A booster will then be required around day 28 of age for carrying the flock through adult period of production [16]. The post vaccination titer level at the age of eight weeks antibody titer level of broiler breeder (Cobb-500) was protective against IBD. Besides at the age of 23 weeks titer level was 7,165 to 10,733 , which is enough to produce good MDA for offspring. This result obtained by applying 4way killed vaccine against the 20 weeks (just before laying) of age. Inactivated vaccines are essentially used to produce high, uniform and persistent antibody titers in hens prior to lay that have been vaccinated with a live virus or have been naturally infected through exposure to the virus on the farm $[17,18]$. These vaccines are administered by the subcutaneous or intramuscular route at the age of 16-20 weeks. Progeny of hens that have been vaccinated in this way have protective antibodies until the age of approximately $30 \mathrm{~d}[19,20]$. The chicks are thus protected during the period of susceptibility to the IBDV strains that only provoke immunosuppression. However, the chicks are not protected from other highly pathogenic strains that may inflict high mortality rates at later stages [20]. The highest titer level of MDA at day one was 4,448 (G.T.M) but Besseboua et al. (2015) [21] noticed that the level was $6,400.54 \pm 2,993.67$ MDA. Drastically fall the MDA level in broiler breeder Cobb-500 at the age of $14 \mathrm{~d}$. IBD antibody titer level at 50 weeks of age groups indicated that the MT at mid laying stage the titer level was enough to produce high MDA for their offspring.

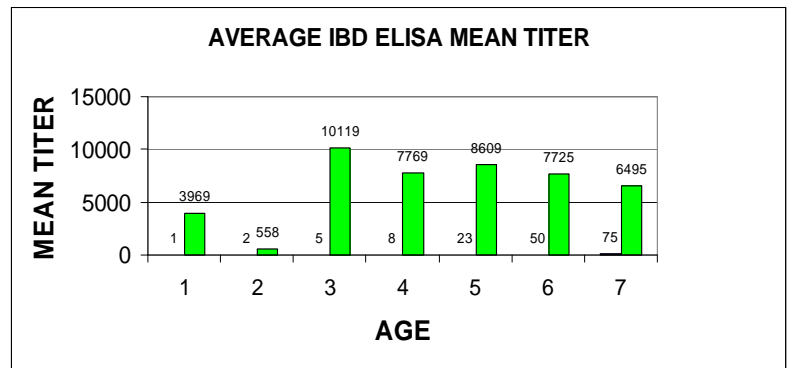

Fig. 5 Average mean titer at different ages (weeks) in bar diagram. 
Table 1 Maternally derived antibody (MDA) level of different flocks at age day one.

\begin{tabular}{llllllll}
\hline Flock & Assay & Age (day) & MT & Min.-Max. & G.M.T. & \% CV & Gr.Ti. range \\
\hline 1 & IBD & 1 & 4,821 & $1,661-7,559$ & 4,448 & 37 & $2-7$ \\
2 & IBD & 1 & 4,771 & $333-8,723$ & 3,962 & 46 & $0-8$ \\
3 & IBD & 1 & 5,184 & $484-9,606$ & 4,224 & 54 & $1-8$ \\
4 & IBD & 1 & 3,395 & $564-6,175$ & 2,979 & 54 & $1-7$ \\
5 & IBD & 1 & 4,582 & $1,920-6,827$ & 4,203 & 37 & $2-7$ \\
\hline
\end{tabular}

MT = mean titer, CV = coefficient of variation, G.M.T. = geometric mean titer, Gr.Ti. = group titer, IBD = infectious bursal disease.

Table 2 MDA level of different flocks at age of $14 \mathrm{~d}$.

\begin{tabular}{llllllll}
\hline Flock & Assay & Age (day) & MT & Min.-Max. & G.M.T. & \% CV & Gr.Ti. range \\
\hline 1 & IBD & 14 & 505 & $57-1,369$ & 360 & 80 & $0-2$ \\
2 & IBD & 14 & 679 & $264-1,637$ & 614 & 51 & $0-2$ \\
3 & IBD & 14 & 536 & $164-1,454$ & 457 & 60 & $0-2$ \\
4 & IBD & 14 & 514 & $1-1,083$ & 315 & 63 & $0-2$ \\
5 & IBD & 14 & 558 & $16-1,752$ & 368 & 83 & $0-2$ \\
\hline
\end{tabular}

MT = mean titer, CV = coefficient of variation, G.M.T. = geometric mean titer, Gr.Ti. = group titer, IBD = infectious bursal disease .

Table 3 Post vaccination titer level at the age five weeks.

\begin{tabular}{llllllll}
\hline Flock & Assay & Age (week) & MT & Min.-Max. & G.M.T. & \% CV & Gr.Ti. range \\
\hline 1 & IBD & 5 & 10,517 & $1,573-13,854$ & 9,472 & 34 & $2-10$ \\
2 & IBD & 5 & 8,539 & $5,570-11,535$ & 8,407 & 17 & $6-9$ \\
3 & IBD & 5 & 10,562 & $7,269-17,748$ & 10,324 & 24 & $7-12$ \\
4 & IBD & 5 & 11,013 & $6,610-19,323$ & 10,632 & 28 & $7-13$ \\
5 & IBD & 5 & 9,966 & $4,226-17,723$ & 9,074 & 44 & $5-12$ \\
\hline
\end{tabular}

MT = mean titer, CV = coefficient of variation, G.M.T. = geometric mean titer, Gr.Ti. = group titer, IBD = infectious bursal disease.

Table 4 Post vaccination titer level at the age eight weeks.

\begin{tabular}{llllllll}
\hline Flock & Assay & Age (week) & MT & Min.-Max. & G.M.T. & \% CV & Gr.Ti. range \\
\hline 1 & IBD & 8 & 7,407 & $5,028-10,376$ & 7,299 & 18 & $6-9$ \\
2 & IBD & 8 & 6,435 & $4,260-10,940$ & 6,267 & 25 & $5-9$ \\
3 & IBD & 8 & 8,238 & $4,765-14,429$ & 7,909 & 30 & $5-11$ \\
4 & IBD & 8 & 8,627 & $3,059-11,992$ & 8,125 & 31 & $4-9$ \\
5 & IBD & 8 & 8,138 & $2,896-14,456$ & 7,266 & 47 & $3-11$ \\
\hline
\end{tabular}

MT = mean titer, $\mathrm{CV}=$ coefficient of variation, G.M.T. = geometric mean titer, Gr.Ti. = group titer, IBD = infectious bursal disease.

Table 5 IBD antibody titer level at 23 weeks of age groups.

\begin{tabular}{llllllll}
\hline Flock & Assay & Age (week) & MT & Min.-Max. & G.M.T. & \% CV & Gr.Ti. range \\
\hline 1 & IBD & 23 & 8,373 & $5,090-10,371$ & 8,226 & 18 & $6-9$ \\
2 & IBD & 23 & 7,816 & $4,730-10,835$ & 7,626 & 22 & $5-9$ \\
3 & IBD & 23 & 7,165 & $115-12,471$ & 5,700 & 43 & $0-10$ \\
4 & IBD & 23 & 10,733 & $7,300-12,312$ & 10,639 & 13 & $7-10$ \\
5 & IBD & 23 & 8,962 & $6,175-11,805$ & 8,813 & 18 & $7-9$ \\
\hline
\end{tabular}

MT = mean titer, CV = coefficient of variation, G.M.T. = geometric mean titer, Gr.Ti. = group titer, IBD = infectious bursal disease. 
Table 6 IBD antibody titer level at 50 weeks of age groups.

\begin{tabular}{llllllll}
\hline Flock & Assay & Age (week) & MT & Min.-Max. & G.M.T. & \% CV & Gr.Ti. range \\
\hline 1 & IBD & 50 & 7,295 & $3,861-11,491$ & 6,916 & 33 & $4-9$ \\
2 & IBD & 50 & 7,250 & $4,108-12,030$ & 6,921 & 32 & $5-10$ \\
3 & IBD & 50 & 7,986 & $4,614-11,039$ & 7,673 & 28 & $5-9$ \\
4 & IBD & 50 & 8,829 & $6,089-12,817$ & 8,609 & 22 & $7-10$ \\
5 & IBD & 50 & 7,265 & $4,649-11,500$ & 7,034 & 26 & $5-9$ \\
\hline
\end{tabular}

MT = mean titer, CV = coefficient of variation, G.M.T. = geometric mean titer, Gr.Ti. = group titer, IBD = infectious bursal disease.

Table 7 IBD antibody titer level at 75 weeks of age groups.

\begin{tabular}{llllllll}
\hline Flock & Assay & Age (week) & MT & Min.-Max. & G.M.T. & \% CV & Gr.Ti. range \\
\hline 1 & IBD & 75 & 7,278 & $3,859-11,529$ & 6,851 & 35 & $4-9$ \\
2 & IBD & 75 & 4,783 & $1,841-8,461$ & 4,511 & 34 & $2-8$ \\
3 & IBD & 75 & 5,997 & $2,278-10,021$ & 5,580 & 37 & $3-9$ \\
4 & IBD & 75 & 7,422 & $3,663-10,446$ & 7,044 & 49 & $4-9$ \\
5 & IBD & 75 & 6,995 & $3,763-9,175$ & 6,809 & 23 & $4-8$ \\
\hline
\end{tabular}

MT = mean titer, CV = coefficient of variation, G.M.T. = geometric mean titer, Gr.Ti. = group titer, IBD = infectious bursal disease.

IBD antibody titer level at 75 weeks of age groups the MT was declined at last stage of laying (just before culling) but the titer level was enough to produce protective MDA for their offspring.

\section{Conclusions}

Timing of administering a live IBD vaccination to chickens with maternal antibodies is often tricky and difficult to do correctly. As maternal antibodies will neutralize the vaccine virus one needs to wait with vaccination until maternal antibody levels are at such a low level, that the vaccination virus will not be neutralized by maternal antibodies (optimal titer to vaccinate). On the other hand, one does not want to wait too long with vaccination as this will leave the flock exposed to field infection. The principle behind predicting is simple; as there is a regular decline of maternal antibodies in the first weeks of life, one can measure antibody levels early in the life of a chick and then predict when the level will be low enough to allow for vaccination (optimal titer to vaccinate).

Most of the broiler breeder industry has adopted the strategy of hyper-immunizing the breeders for IBD. By providing a series of live vaccines prior to the killed injections, it could attempt to produce high uniform maternal antibodies, which will pass to the chicks. The amount of protection the chicks receive via the yolk from the mother is directly proportional to the level of immunity of the mother, all other things being equal. Extreme care must be taken to ensure the vaccines are handled properly and delivered appropriately so that each bird receives an immunizing dose. Strict attention to detail can mean the difference between protected birds and those that remain susceptible. It is important to read the information supplied with the vaccine vials, administer it properly and pay close attention to withdrawal times. Administration of vaccines properly at the correct time and with the appropriate antigen content does not guarantee $100 \%$ protection. However, a well-designed, well timed and soundly executed vaccination program coupled with good management, nutrition and biosecurity will go a long way to helping maintain a healthy and productive flock. Current study will help in term of preparation of vaccination baseline for broiler breeder and vaccination schedule for (offspring) commercial broiler.

\section{Acknowledgments}

The authors would like to thank faculty and stuffs of Department of Microbiology, Hajee Mohammad Danesh Science and Technology University, 


\section{Quantification of Protective Antibody Vaccine-Elicited in Chickens against Infectious Bursal Disease Virus}

Dinajpur-5200, Bangladesh for support.

\section{References}

[1] Jordan, F. T. W., and Pattison, M. 1996. "Infectious Bursal Disease.” In Poultry Diseases, 5th ed., 205-15.

[2] Toro, H., Van Santen, V. L., Hoerr, F. J., and Breedlove, C. 2009. "Effects of Chicken Anemia Virus and Infectious Bursal Disease Virus in Commercial Chickens.” Avian Dis. 53: 94-102.

[3] Jackwood, D. J., and Sommer-Wagner, S. E. 2010. "Detection and Characterization of Infectious Bursal Disease Viruses in Broilers at Processing." Prev. Vet. Med. 97 (1): 45-50.

[4] Jackwood, D. J., and Saif, Y. M. 1987. "Antigenic Diversity of Infectious Bursal Disease Viruses.” Avian Dis. 31: 766-70.

[5] Cosgrove, A. S. 1962. "An Apparently New Disease of Chickens-Avian Nephrosis.” Avian Dis. 6: 385-9.

[6] Shrestha, P., Ahsan, M. M., Islam, K. M. D., Billah, M. M., Mehedi, M., Mitre, S., and Islam, M. R. 2003. "Seroprevalence of Infectious Bursal Disease Virus (IBDV) Specific Antibody in Chicken.” Pak. J. of Biological Sci. 6 (14): 1234-40.

[7] Cho, Y., and Edgar, S. A. 1972. "Characterization of Infectious Bursal Disease.” Poult. Sci. 51: 60-9.

[8] Giambrone, J. J. 1987. "Infectious Bursal Disease Virus Variants Termed Emerging Problem.” Poult. Digest. 46: 116-20.

[9] Vandersluis, W. 1999. "World Poultry Disease Update.” World Poult. 15: 30-3.

[10] De Wit, J. J. 2001. "Gumboro Disease: Estimation of Optimal Time of Vaccination by the Deventer Formula." In Annual Report and Proceedings of COST Action 839: Immunosuppressive Viral Diseases in Poultry, 170-8.

[11] Jung, T.-H., Lin-DihFa, and Sung, H. T. 1995. "Survey of Maternal Antibody Status against IBDV and Estimation of the Vaccination Point.” J. Chinese Soc. Vet. Sci. 21: 223-31.

[12] Saijo, K., and Higashihara, M. 1998. "Optimal Time of Initial Administration of Live Vaccine for IBD in Chicks with MDA.” J. Japan Vet. Med. Asso. 51: 647-51.

[13] Al-Natour, M. Q., Ward, L. A., Saif, Y. M., Stewart-Brown, B., and Keck, L. D. 2004. "Effect of Different Level of Maternally Derived Antibodies on Protection against Infectious Bursal Disease Virus." Avian Dis. 48 (1): 177-82.

[14] Azab, A., Hassan, S. M., and Qubih, T. S. 1991. "Determination of Maternal Immunity against IBD in Broiler Chicks.” Assiut. Vet Med. J. 25: 211-5.

[15] Wisniewska, J., and Stosik, M. 1999. "Serum Antibody Titer after the First Immunization of Broilers against IBDV.” Medycyna Waterynaryjna 55: 48-51.

[16] Zaheer, A., and Saeed, A. 2003. "Role of Maternal Antibodies in Protection against Infectious Bursal Disease in Commercial Broilers.” Int. J. of Poultry Sci. 2 (4): 251-5.

[17] Cullen, G. A., and Wyeth, P. J. 1976. "Response of Growing Chickens to an Inactivated IBD Antigen in Oil Emulsion.” Vet. Rec. 99: 418.

[18] Guittet, M., Le, C. H., Picault, J. P., Eterradossi, N., and Bennejean, G. 1992. "Safety of Infectious Bursal Disease Vaccines: Assessment of an Acceptability Threshold." Dev. Biol. Standard. 79: 147-52.

[19] Box, P. 1989. "High Maternal Antibodies Help Chickens Beat Virulent Virus.” World Poultry 53: 17-9.

[20] Van den Berg, T. P., and Meulemans, G. 1991. "Acute Infectious Bursal Disease in Poultry: Protection Afforded by Maternally Derived Antibodies and Interference with Live Vaccination.” Avian Pathol 20 (3): 409-21.

[21] Besseboua, O., Ayad, A., and Benbarek, H. 2015. "Determination of the Optimal Time of Vaccination against Infectious Bursal Disease Virus (Gumboro) in Algeria." Onderstepoort Journal of Veterinary Research 82 (1): 887. 